\title{
Investigations of the EPR and Optical Spectra for $\mathrm{VO}^{2+}$ in $\mathrm{C}_{3} \mathrm{H}_{7} \mathrm{NO}_{2}$ Powders
}

\author{
Q. WEI* \\ Department of Physics, Xi'dian University, Xi'an 710071, P.R. China \\ (Received December 31, 2009; in final form April 19, 2010)
}

\begin{abstract}
The EPR and optical spectra for $\mathrm{VO}^{2+}$ in $\mathrm{C}_{3} \mathrm{H}_{7} \mathrm{NO}_{2}$ powders are calculated from complete diagonalization method and perturbation theory method, respectively. The calculated results are in good agreement with observed values. The negative signs of hyperfine structure constants $A_{\|}$and $A_{\perp}$ for $\mathrm{VO}^{2+}$ in $\mathrm{C}_{3} \mathrm{H}_{7} \mathrm{NO}_{2}$ powders are also suggested from the calculations.
\end{abstract}

PACS numbers: $76.30 \mathrm{Fc}, 71.70 \mathrm{Ch}, 78.50 \mathrm{Ec}, 71.55 \mathrm{Ht}$

\section{Introduction}

The $\mathrm{VO}^{2+}\left(\right.$ or $\left.\mathrm{V}^{4+}\right)$ ions were used extensively as a dopant material in various systems [1-9]. Recently, $\mathrm{VO}^{2+}$ doped $\mathrm{C}_{3} \mathrm{H}_{7} \mathrm{NO}_{2}$ powders have been examined by electron paramagnetic resonance (EPR) and optical absorption spectroscopy [5]. The experimental values of $g$ factors are $g_{\|}=1.932$ and $g_{\perp}=1.999$. As known, an octahedral site with a tetragonal compression would give the value of $g_{\|}<g_{\perp}<g_{e}$, where $g_{e}$ is the free-spin $\mathrm{g}$ value of 2.0023 . The observed values of the spin Hamiltonian parameters agree with the relation. That is to say, $\mathrm{VO}^{2+}$ complexes in $\mathrm{C}_{3} \mathrm{H}_{7} \mathrm{NO}_{2}$ powders are tetragonally distorted octahedral symmetry. Until now, no satisfactory theoretical explanations for the optical and EPR parameters have been made. In this paper, the optical and EPR spectra have been theoretical explained by using perturbation theory method (PTM) and complete diagonalization method (CDM), respectively. And the crystal field (CF) parameters $D q, D s$ and $D t$ are obtained.

\section{Calculations}

\subsection{Perturbation theory method}

$\mathrm{V}^{4+}$ is of the $3 d^{1}$ type. There is only one term for free $3 d^{1}$ ions, i.e. ${ }^{2} D$ term. ${ }^{2} D$ term is split by an octahedron CF $\left(O_{h}\right)$ into ${ }^{2} E_{g}$ and ${ }^{2} T_{2 g}$ terms. If the octahedron is distorted along the tetragonal axis, the symmetry is lowered. Then the ${ }^{2} E_{g}$ term splits to ${ }^{2} A_{1}$ and ${ }^{2} B_{1}$, the ${ }^{2} T_{2 g}$ splits to ${ }^{2} B_{2}$ and ${ }^{2} E$. In compressed tetragonal octahedral symmetry, the ground state is ${ }^{2} B_{2}$. Thus, the energy

* corresponding author; e-mail: Weiaqun@163.com intervals can be expressed as:

$$
\begin{aligned}
& E_{1}=E\left({ }^{2} E\right)-E\left({ }^{2} B_{2}\right)=-3 D s+5 D t, \\
& E_{2}=E\left({ }^{2} B_{1}\right)-E\left({ }^{2} B_{2}\right)=10 D q, \\
& E_{3}=E\left({ }^{2} A_{1}\right)-E\left({ }^{2} B_{2}\right)=10 D q-4 D s-5 D t,
\end{aligned}
$$

where $D q$ is the cubic crystal field parameter, $D s$ and $D t$ are the tetragonal crystal field parameters.

For $3 d^{1}$ ions in tetragonal $\mathrm{CF}$ the effective spin-Hamiltonian ( $\mathrm{SH})$ is given as [3]:

$$
\begin{aligned}
& H_{\mathrm{S}}=g_{\|} \mu_{\mathrm{B}} B_{z} S_{z}+g_{\perp} \mu_{\mathrm{B}}\left(B_{x} S_{x}+B_{y} S_{y}\right)+A_{\|} I_{z} S_{z} \\
& \quad+A_{\perp}\left(I_{x} S_{x}+I_{y} S_{y}\right) .
\end{aligned}
$$

The symbols appearing in Eq. (4) have their usual meanings. $g_{\|}$and $g_{\perp}$ are the parallel and perpendicular components of the g-tensor. $A_{\|}$and $A_{\perp}$ are the parallel and perpendicular components of the hyperfine tensor. The third-order perturbation formulas of spin-Hamiltonian parameters for $3 d^{1}$ ions in tetragonal symmetry with the ground state ${ }^{2} B_{2}$ can be derived from the perturbation theory as [9]:

$$
\begin{aligned}
& g_{\|}=g_{e}-\frac{8 k \zeta}{E_{2}}-\frac{\left(k+g_{e}\right) \zeta^{2}}{E_{1}^{2}}-\frac{4 k \zeta^{2}}{E_{1} E_{2}}, \\
& g_{\perp}=g_{e}-\frac{2 k \zeta}{E_{1}}+\frac{\left(k-g_{e}\right) \zeta^{2}}{E_{1}^{2}}-\frac{2 g_{e} \zeta^{2}}{E_{2}^{2}}, \\
& A_{\|}=P\left[-\kappa-\frac{4}{7}+\left(g_{\|}-g_{e}\right)+\frac{3}{7}\left(g_{\perp}-g_{e}\right)\right], \\
& A_{\perp}=P\left[-\kappa+\frac{2}{7}+\frac{11}{14}\left(g_{\perp}-g_{e}\right)\right],
\end{aligned}
$$

where $k$ is the orbital-reduction factor, we take it as 0.7 here. $E_{1}$ and $E_{2}$ are the energy intervals given in Eqs. (1) and (2). $\kappa$ is the core polarization constant which indicates the contribution to the $A_{\|}$and $A_{\perp}$ by the unpaired s-electron. $P$ is the dipolar hyperfine structure constant 
for $\mathrm{VO}^{2+}$ in crystals. Considering the covalence reduction effect, $\zeta$ and $P$ can be calculated from the relations $[9-11]:$

$$
\zeta \approx k \zeta_{0}, P \approx k P_{0},
$$

here $\zeta_{0}=248 \mathrm{~cm}^{-1}[12]$ and $P_{0}=172 \times 10^{-4} \mathrm{~cm}^{-1}$ [13] are the values of $\zeta$ and $P$ of $\mathrm{V}^{4+}$ in free state. By fitting the observed EPR and optical spectra, we obtained the value of CF parameters are: $D q=1466 \mathrm{~cm}^{-1}$, $D s=-2804 \mathrm{~cm}^{-1}, D t=637 \mathrm{~cm}^{-1}$. Substituting these CF parameters into above formulas, the energy levels and EPR parameters can be calculated. The calculated results are listed in the Table.

TABLE

The EPR and optical spectra for $\mathrm{VO}^{2+}$ in $\mathrm{C}_{3} \mathrm{H}_{7} \mathrm{NO}_{2}$ powders. (The units of optical spectra are in $\mathrm{cm}^{-1}$, the units of $A_{\|}$and $A_{\perp}$ are in $10^{-4} \mathrm{~cm}^{-1}$.)

\begin{tabular}{c|c|c|c}
\hline \hline & $\begin{array}{c}\text { Calculated } \\
\text { by PTM }\end{array}$ & $\begin{array}{c}\text { Calculated } \\
\text { by CDM }\end{array}$ & $\begin{array}{c}\text { Observed } \\
\text { value [5] }\end{array}$ \\
\hline${ }^{2} B_{2} \rightarrow{ }^{2} E$ & 11597 & 11510 & 11595 \\
& & 11684 & \\
${ }^{2} B_{2} \rightarrow{ }^{2} B_{1}$ & 14660 & 14670 & 14658 \\
${ }^{2} B_{2} \rightarrow{ }^{2} A_{1}$ & 22691 & 22698 & 22696 \\
$g_{\|}$ & 1.9349 & 1.9349 & 1.932 \\
$g_{\perp}$ & 1.9805 & 1.9807 & 1.999 \\
$A_{\|}$ & -182.8 & -182.8 & 187.0 \\
$A_{\perp}$ & -72.4 & -72.4 & 72.1
\end{tabular}

\subsection{Complete diagonalization method}

The energy matrices for the $d^{1}$ configuration ion with tetragonal symmetry have been established based on the following Hamiltonian:

$$
H=H_{f}+H_{\mathrm{CF}}(D q, D s, D t)+H_{\mathrm{SO}}(\zeta)
$$

where $H_{f}$ and $H_{\mathrm{SO}}$ are the free-ion term and spin-orbit coupling interaction, respectively. $\zeta$ is the spin-orbit coupling constant. The crystal field interaction term can be written as:

$$
H_{\mathrm{CF}}=B_{20} C_{0}^{(2)}+B_{40} C_{0}^{(4)}+B_{44} C_{4}^{(4)}+B_{4-4} C_{-4}^{(4)}
$$

where $C_{q}^{(k)}=\sqrt{\frac{4 \pi}{2 k+1}} Y_{k q}$ are normalized spherical harmonics, and $B_{k q}$ are CF parameters with $B_{44}=B_{4-4}$. The CF parameter $B_{k q}$ measure the strength of the interaction between the open-shell electrons of the paramagnetic ions and their surrounding crystalline environment. they can be expressed as:

$$
\begin{aligned}
& B_{20}=-7 D s, \\
& B_{40}=21 D q-21 D t, \\
& B_{44}=21 \sqrt{\frac{5}{14}} D q .
\end{aligned}
$$

Our calculations for the Hamiltonian matrices are carried out in the intermediate crystal field coupling scheme [14]. In the intermediate crystal field coupling scheme, the eigenfunctions have the form $\left|S L M_{\mathrm{S}} M_{\mathrm{L}}\right\rangle$. According to the group theory, one can obtain all the 10 eigenfunctions of $d^{1}$ ions in tetragonal symmetry. The matrix element of the crystal field $H_{\mathrm{CF}}$ and the spin-orbit coupling $H_{\mathrm{SO}}$ can be expressed as:

$$
\begin{aligned}
& \left\langle d^{1} S L M_{\mathrm{S}} M_{\mathrm{L}}\left|H_{\mathrm{CF}}\right| d^{1} S^{\prime} L^{\prime} M_{\mathrm{S}}^{\prime} M_{\mathrm{L}}^{\prime}\right\rangle=\delta_{\mathrm{M}_{\mathrm{S}} \mathrm{M}_{\mathrm{S}}^{\prime} \delta_{\mathrm{SS}^{\prime}}} \\
& \times \sum_{k, q} B_{k q}\left\langle d|| C^{(k)} \| d\right\rangle\left[\begin{array}{ccc}
L & k & L^{\prime} \\
-M_{\mathrm{L}} & q & M_{\mathrm{L}}^{\prime}
\end{array}\right] \\
& \quad \times\left\langle d^{1} S L\left\|U^{(k)}\right\| d^{1} S^{\prime} L^{\prime}\right\rangle, \\
& \left\langle d^{1} S L M_{\mathrm{S}} M_{\mathrm{L}}\left|H_{\mathrm{SO}}\right| d^{1} S^{\prime} L^{\prime} M_{\mathrm{S}}^{\prime} M_{\mathrm{L}}^{\prime}\right\rangle \\
& =\left\langle d\left\|l^{(1)}\right\| d\right\rangle \zeta \sum_{q=-1}^{1}(-1)^{q+S+L-M_{\mathrm{S}}-M_{\mathrm{L}}} \\
& \times\left[\begin{array}{ccc}
S & 1 & S^{\prime} \\
-M_{\mathrm{S}} & -q & M_{\mathrm{S}}^{\prime}
\end{array}\right]\left[\begin{array}{rrr}
L & 1 & L^{\prime} \\
-M_{\mathrm{L}} & q & M_{\mathrm{L}}^{\prime}
\end{array}\right] \\
& \times\left\langle d^{1} S L\left\|V^{(11)}\right\| d^{1} S^{\prime} L^{\prime}\right\rangle,
\end{aligned}
$$

where the [ ] indicates $3 \mathrm{j}$-symbols. The reduced matrix elements and the electrostatic matrix elements can be found in [15].

By means of the equivalence between the spin Hamiltonian and the Zeeman interaction, the $\mathrm{g}$ factors can be expressed as [9]:

$$
\begin{aligned}
& g_{\|}=2\left\langle\psi_{+}\left|k L_{z}+g_{e} S_{z}\right| \psi_{+}\right\rangle, \\
& g_{\perp}=2\left\langle\psi_{+}\left|k L_{x}+g_{e} S_{x}\right| \psi_{-}\right\rangle,
\end{aligned}
$$

where $\left|\psi_{+}\right\rangle$and $\left|\psi_{-}\right\rangle$express the two full configuration eigenfunctions of eigenstates ${ }^{2} B_{2}$, and the detailed expressions used in the present paper are listed in the appendix. The expressions of $A_{\|}$and $A_{\perp}$ are the same as those in Eqs. (7) and (8).

Substituting the CF parameters into Eqs. (10)-(16), one can obtain the $10 \times 10$ complete energy matrix, After diagonalizing the matrix, the energy levels and the eigenfunctions of ground state can be obtained. The eigenfunctions can be used to calculate the $g$ factors. The calculated results are also listed in the Table.

\section{Discussion and conclusion}

(1) One can see from the Table, that results obtained by CDM and PTM are not only very close to each other, but also in good agreement with observed values. Thus, the EPR and optical spectra of $\mathrm{VO}^{2+}$ in $\mathrm{C}_{3} \mathrm{H}_{7} \mathrm{NO}_{2}$ powders are explained theoretically. In CDM, all the microscopic states are considered, and the results calculated from CDM can be regarded as accurate results. On this basis, some PTM formulas are checked [16-18]. The validity of PTM formulas of $g$-factors for $3 d^{1}$ ions at tetragonal symmetry have been investigated in [16]. It is shown, that the PTM formulas are reasonable and reliable in a wide range of $\mathrm{CF}$ parameters. From Eqs. (1)-(3), (5) and (6), one can see, that only the highest energy level ${ }^{2} A_{1}$ is omitted in these third-order 
perturbation formulas. So the results calculated by PTM can agree well with those by CDM.

(2) It should be noted, that the signs of $A_{\|}$and $A_{\perp}$ for $3 d^{n}$ or $4 f^{n}$ ions in crystals can not be determined from EPR spectra directly. So, the experimental values of $A_{\|}$and $A_{\perp}$ are actually the absolute values. Muncaster and Parke [19] have proved that the signs of $A_{\|}$and $A_{\perp}$ should be negative for $\mathrm{VO}^{2+}$ ions in hydrated salts and glasses. In this paper, we found, that the signs of $A_{\|}$ and $A_{\perp}$ for $\mathrm{VO}^{2+}$ in $\mathrm{C}_{3} \mathrm{H}_{7} \mathrm{NO}_{2}$ powders also should be negative. This is coincident with the $\mathrm{VO}^{2+}$ in other crystals [9].

(3) The optical absorption spectrum shows three bands characteristic of $\mathrm{VO}^{2+}$ ions in tetragonal symmetry. The cubic crystal field parameter $D q$ and the tetragonal field parameters $D s$ and $D t$ were obtained. The values of $\mathrm{CF}$ parameters we obtained are: $D q=1466 \mathrm{~cm}^{-1}, D s=$ $-2804 \mathrm{~cm}^{-1}, D t=637 \mathrm{~cm}^{-1}$. These values are similar as $\mathrm{VO}^{2+}$ in other crystals $[20,21]$. So, the CF parameters we obtained in this paper are reliable.

\section{Acknowledgments}

This work was supported by the Education Committee Natural Science Foundation of Shaanxi Province (Grant No. 08JK216), a Baoji University of Arts and Sciences Key Research Grant (Grant No. ZK 0713), and HPC Lab, Shenzhen Institute of Advanced Technology, CAS, China.

\section{Appendix}

The two full configuration eigenfunctions of eigenstates ${ }^{2} B_{2},\left|\psi_{+}\right\rangle$and $\left|\psi_{-}\right\rangle$, are as follows:

$$
\begin{aligned}
& \left|\psi_{+}\right\rangle=0.999866 \frac{1}{\sqrt{2}}\left(\left|2,2, \frac{1}{2}, \frac{1}{2}\right\rangle-\left|2,-2, \frac{1}{2}, \frac{1}{2}\right\rangle\right) \\
& -0.011741 \frac{1}{\sqrt{2}}\left(\left|2,2, \frac{1}{2}, \frac{1}{2}\right\rangle+\left|2,-2, \frac{1}{2}, \frac{1}{2}\right\rangle\right) \\
& \left|\psi_{-}\right\rangle=0.999866 \frac{1}{\sqrt{2}}\left(\left|2,2, \frac{1}{2},-\frac{1}{2}\right\rangle-\left|2,-2, \frac{1}{2},-\frac{1}{2}\right\rangle\right) \\
& +0.011741 \frac{1}{\sqrt{2}}\left(\left|2,2, \frac{1}{2},-\frac{1}{2}\right\rangle+\left|2,-2, \frac{1}{2},-\frac{1}{2}\right\rangle\right)
\end{aligned}
$$

\section{References}

[1] N.O. Gopal, K.V. Narasimhulu, J.L. Rao, Physica B 307, 117 (2001).

[2] Y. Yerli, F. Koksal, A. Karadag, Solid State Sci. 54, 1319 (2003).

[3] H. Farah, J. Mater. Sci. 38, 727 (2003).

[4] K.V. Narasimhulu, J.L. Rao, Spectrochim. Acta A 53, 2605 (1997)

[5] R. Biyik, Physica B 404, 3483 (2009).

[6] V.K. Jain, Phys. Stat. Sol. B 97, 337 (1980).

[7] B. Karabulut, I. Ilkin, R. Tapramaz, Z. Naturforsch. 60a, 95 (2005).

[8] B. Karabulut, A. Tufan, Spectrochim. Acta A 65, 742 (2006).

[9] F. Wang, W.C. Zheng, L. He, Spectrochim. Acta A 71, 513 (2008).

[10] M.G. Zhao, J.A. Xu, G.R. Bai, H.S. Xie, Phys. Rev. B 27, 1516 (1983).

[11] W.C. Zheng, Q. Zhou, X.X. Wu, Y. Mei, Z. Naturforsch. 61a, 286 (2006).

[12] J.S. Griffith, The Theory of Transition-Metal Ions, Cambridge University Press, London 1964.

[13] B.R. McGarvey, J. Phys. Chem. 71, 51 (1967).

[14] W.L. Yu, X.M. Zhang, L.X. Yang, B.Q. Zen, Phys. Rev. B 50, 6756 (1994).

[15] C.W. Nielson, G.F. Koster, Spectroscopic Coefficients for the $p^{n}, d^{n}$, and $f^{n}$ Configurations, MIT Press, Cambridge 1963.

[16] Q. Wei, Pramana J. Phys. 73, 1087 (2009).

[17] Y. Hao, Z.Y. Yang, J. Magn. Magn. Mater. 299, 445 (2006).

[18] Q. Wei, Chin. J. Phys. 46, 181 (2008).

[19] R. Muncaster, S. Parke, J. Non-Cryst. Solids 24, 399 (1977).

[20] R. Kripal, M. Maurya, H. Govind, Physica B 392 , 281 (2007).

[21] R. Kripal, I. Mishra, S.K. Gupta, M. Arora, Spectrochim. Acta A 71, 1969 (2009). 